\title{
Pulse Oximeter Perfusion Index as a Predictor of Successful Supraclavicular Brachial Plexus Block
}

\author{
Veena $^{1} \quad$ Karthik S. L. ${ }^{1} \quad$ Vishwanath Ankad ${ }^{2}$ \\ ${ }^{1}$ Department of Anaesthesiology, Najappa Hospital, Shivamogga, \\ Karnataka, India \\ ${ }^{2}$ Department of Anaesthesiology, ESIC Medical College, Bangalore, \\ Karnataka, India
}

\begin{abstract}
Address for correspondence Veena, DA, DNB, Sri Benaka, 2 Cross, B Block, Church Main Road, Sharavathi Nagar, Shivamogga, 577201 Karnataka, India (e-mail: veenadoc61@gmail.com).
\end{abstract}

Int J Recent Surg Med Sci 2022;8:18-21.

\begin{abstract}
Keywords

- supraclavicular brachial plexus block

- perfusion index

- bupivacaine

- ultrasound guide

- perfusion index ratio

Objective Ultrasound-guided supraclavicular brachial plexus block is a popular anesthetic technique for upper limb surgeries. Assessing the success sensory and motor block using conventional methods is time consuming and also it needs patient co-operation. In the present study, objective method like increase in perfusion index is used to predict the success of ultrasound-guided supraclavicular block.

Materials and Methods This prospective trial consists of total 95 patients undergoing elective upper limb procedures. All patients received ultrasound-guided supraclavicular block using $0.5 \%$ bupivacaine $20 \mathrm{~mL}$. Sensory and motor blocks were evaluated every 5 minutes followed by pinprick testing and ability to flex the elbow and the hand against gravity, respectively. The perfusion index was measured using pulse oximetry applied on the index finger and recorded at baseline and 10, 20, and 30 minutes interval after local anesthetic injection in both the blocked limb and the contralateral unblocked limb using two separate pulse oximeters.

Results Perfusion index increased in blocked arm after 5 minutes compared with unblocked arm and also to its baseline value. Both perfusion index and perfusion index ratio in blocked arm were found statistically significant.

Conclusion To evaluate the success of supraclavicular block, perfusion index can be considered as a useful tool.
\end{abstract}

\section{Introduction}

Ultrasound-guided supraclavicular nerve block is a popular approach for anesthesia for upper limb surgeries. The success of peripheral nerve blocks is usually evaluated by assessment of sensory and motor function; however, this method is subjective, time consuming, and depends on patient co-operation. ${ }^{1}$ Various objective methods for evaluation of block success have been developed. ${ }^{2-4}$ Objective methods for block assessment depend on the evaluation of the sympathetic block and consequent physiological changes,

DOI https://doi.org/

$10.1055 / \mathrm{s}-0041-1734211$

ISSN 2455-7420 such as vasodilation and changes in blood flow ${ }^{2,3}$ and skin temperature. ${ }^{4}$ However, most of the objective methods are either time consuming or dependent on sophisticated equipment.

The perfusion index (PI) is a numerical value for the ratio between pulsatile and nonpulsatile blood flow measured by a pulse oximeter. PI which is automatically calculated by pulse oximetry and provides an indication of peripheral perfusion at the sensor site (finger) has been shown to be a useful method to evaluate axillary or sciatic block in patients scheduled for limb surgery. ${ }^{5,6}$ 
There are limited studies available to show increase in PI as a tool for evaluation of peripheral block success. ${ }^{7}$ Hence, an attempt was made through this study to evaluate the increase in PI and PI ratio as predictors of successful supraclavicular nerve block in comparison to neurological assessment.

\section{Materials and Methods}

This prospective observational study was undertaken in a tertiary care center, after approval from institutional research and ethics committee. Following a detailed preanesthetic checkup, informed written consent was obtained from patients with following inclusion criteria; American Society of Anesthesiologists (ASA) physical status I or II, age 18 to 60 years of either gender, elective surgical procedures on the upper limb.

Patients with ASA physical status III and IV with history of significant neurological, psychiatric and neuromuscular, alcoholism, drug abuse, on adrenergic receptor agonist or antagonist therapy, with known hypersensitivity to local anesthetic drugs, bleeding disorders, uncontrolled diabetes mellitus, and pregnant women were excluded.

All 95 patients were advised to remain nil per oral for 8 hours. Before performing the procedure 18-gauze venous cannula was secured in opposite hand and routine monitors like pulse oximetry, noninvasive blood pressure, electrocardiogram were attached, and baseline readings were noted. Injection midazolam, intravenous (IV) $1 \mathrm{mg}$ was given to all patients.

The procedure was done by same anesthesiologist to overcome various access approaches, patient position, technique and individual expertise. Procedure was done in supine position with arms adducted. Ultrasound-guided supraclavicular brachial plexus block was performed under all aseptic precautions with 22-gauge echogenic needle using linear probe 8 to $12 \mathrm{~Hz}$ of ultrasound machine (MINDRAY M7). Patients

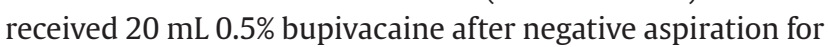
blood/air.

The limb was evaluated for block success every 5 minutes. for the sensory block and motor block. Sensory function was assessed using pinprick in the dermatomal areas supplied by the four main nerves (median nerve, radial nerve, ulnar nerve, and musculocutaneous nerve). Motor block was

Table 1 Demographic characteristics

\begin{tabular}{|l|l|}
\hline Characteristics & Values (Mean \pm SD) \\
\hline Age (years) & $39.28 \pm 12.34$ \\
\hline Weight $(\mathrm{kg})$ & $61.80 \pm 8.54$ \\
\hline Height $(\mathrm{cm})$ & $163.30 \pm 10.04$ \\
\hline Gender (M/F) & $54 / 41$ \\
\hline Hemoglobin (g\%) & $10.78 \pm 1.02$ \\
\hline Mean heart rate (beats/min) & $61 \pm 17.06$ \\
\hline $\begin{array}{l}\text { Mean systolic blood pressure } \\
\text { (mm Hg) }\end{array}$ & $102 \pm 19.97$ \\
\hline $\begin{array}{l}\text { Mean diastolic blood pressure } \\
(\mathrm{mm} \mathrm{Hg})\end{array}$ & $54 \pm 17.59$ \\
\hline
\end{tabular}

assessed by the ability to flex the elbow and the hand against gravity. The supraclavicular block was considered successful with regard to neurological examination if brachial plexus dermatomes (C5-T1) were completely blocked.

Patient's hemodynamic variables like heart rate (HR), systolic blood pressure, diastolic blood pressure, and oxygen saturation (SPO2) were monitored every 5 minutes. HR of less than 60 considered as bradycardia and treated with atropine $0.6 \mathrm{mg}$ IV Blood pressure of less than $20 \%$ of baseline considered as hypotension and treated with mephentermine $6 \mathrm{mg}$ IV bolus along with IV fluids.

PI was measured using pulse oximetry applied on the index finger. The PI was recorded at baseline and at $0,5,10$, 20 , and 30 minutes after local anesthetic injection in both the blocked limb and the contralateral unblocked limb using two separate pulse oximeters. PI ratio, i.e., PI at 10 minutes to PI at baseline was also calculated.

\section{Statistical Analysis}

Sample size calculated was 84, from a study of Kingslin, ${ }^{7}$ assuming 95\% confidence interval, the power of study of $80 \%$ and $\alpha$ of 0.05 . On anticipating $10 \%$ dropout, 95 patients were included in our trial.

All data were analyzed with SPSS version 21.0 software (IBM Corp. released 2015. IBM SPSS Statistics for Windows, Version 23.0; Armonk, New York, United States). Continuous variables were presented as mean and standard deviation. Student " $t$ " test was used as a text of significance to see the association considering $p$-value $<0.05$ was considered as significant.

\section{Results}

In total, 95 patients were included in the study. Demographic and hemodynamic characteristics were presented in - Table 1. Baseline PI is comparable $(p>0.05)$ in both blocked and unblocked arm ( - Table 2). After 5 minutes of local anesthetic injection PI increased in blocked arm compared with baseline value. PI is statistically significant in blocked arm at 5 minutes of local anesthetic application compared with unblocked arm. There were highly significant association in PI values among blocked and unblocked $\operatorname{arm}(p<0.001)$ at 10 , 20 , and 30 minutes interval. PI ratio (at 10 minutes and at baseline) was found to be highly significant ( $p<0.001)$ among blocked arm compared with unblocked arm.

\section{Discussion}

Various objective methods have been investigated in the literature like resistance index, thermographic temperature measurement, skin temperature, finger photo-plethysmography, noninvasive blood hemoglobin assessment, and the variability index..$^{8-11}$

The PI was previously investigated for evaluation of different conditions of vasodilation, such as induction of anesthesia, ${ }^{12}$ epidural block, ${ }^{13}$ stellate ganglion block, ${ }^{14}$ and successful thoracic sympathectomy. ${ }^{15}$ PI use was previously reported in few studies to know the success of infraclavicular brachial 
Table 2 Perfusion index in blocked and unblocked arm

\begin{tabular}{|l|l|l|l|}
\hline Time interval (min.) & $\begin{array}{l}\text { PI in blocked arm } \\
\text { (Mean } \pm \text { SD) }\end{array}$ & $\begin{array}{l}\text { PI in unblocked arm } \\
\text { (Mean } \pm \text { SD) }\end{array}$ & $p$-Value \\
\hline Baseline & $1.97 \pm 1.02$ & $2.01 \pm 1.03$ & $>0.05$ \\
\hline 5 & $4.62 \pm 1.72$ & $2.72 \pm 1.14$ & $<0.01$ \\
\hline 10 & $7.46 \pm 1.10$ & $2.10 \pm 1.03$ & $<0.001$ \\
\hline 20 & $7.14 \pm 1.53$ & $2.80 \pm 1.50$ & $<0.001$ \\
\hline 30 & $7.70 \pm 1.04$ & $2.70 \pm 0.98$ & $<0.001$ \\
\hline PI ratio & $3.70 \pm 1.07$ & $1.04 \pm 1.00$ & $<0.001$ \\
\hline
\end{tabular}

plexus block, ${ }^{7}$ axillary brachial plexus block, ${ }^{6}$ interscalene brachial plexus block, ${ }^{15}$ and sciatic nerve block. ${ }^{6}$

Vasodilation takes place after a successful supraclavicular brachial plexus block. ${ }^{3}$ Relative increase in pulsatile flow was found in vasodilation after a successful supraclavicular brachial plexus block thereby leading to increase in PI, as PI is a ratio of pulsatile and nonpulsatile components of peripheral blood flow. The PI can therefore be considered as an objective measure for peripheral perfusion that can predict peripheral block success. ${ }^{13}$

In present study PI increased after 5 minutes in blocked arm compared with unblocked arm, which was found to be significant $(p<0.05)$. PI was also increased compared with baseline value in blocked arm. These results were comparable in a study conducted by Sebastiani et $a l,{ }^{16}$ where PI ratio 10 minutes to baseline was found to be significant. Another study conducted by Abdelnasser et al, ${ }^{17}$ revealed that PI was higher in blocked arm at all time points and PI ratio was also found to be higher compared with unblocked limb. Both PI and PI ratio, at 10 minutes after injection, showed a sensitivity and specificity of $100 \%$ for block success. They concluded that PI is a useful tool for the evaluation of successful supraclavicular brachial plexus block. PI ratio in our study was 3.70 \pm 1.07 , whereas in Abdelnasser et al, ${ }^{17}$ mean PI ratio was $2.5 \pm$ 0.4 , the changes in values might be because of the difference in local anesthetic volume and drug use.

The PI is characterized by being simple, rapid and user friendly compared with other objective methods for evaluation of block success. Early and accurate detection of peripheral block success would enable rapid corrective action either by block supplementation or by switching to general anesthesia, this would save the operating room time and improve patient satisfaction. Objective methods of block success would also avoid excessive patient pinpricking and allow block evaluation in sedated and anaesthetized patients.

\section{Conclusion}

PI can be used as a reliable indicator for assessing the success of ultrasound-guided supraclavicular block. But this needs to be confirmed by meta-analytical studies to reach a definitive cut-off value for different techniques (ultrasound vs. nerve stimulator) and drugs (bupivacaine and lidocaine vs. ropivacaine).

\section{Conflict of Interest}

None declared.

\section{References}

1 Curatolo M, Petersen-Felix S, Arendt-Nielsen L. Sensory assessment of regional analgesia in humans: a review of methods and applications. Anesthesiology 2000;93(6):1517-1530

2 Smith GB, Wilson GR, Curry CH, et al. Predicting successful brachial plexus block using changes in skin electrical resistance. Br J Anaesth 1988;60(6):703-708

3 Sørensen J, Bengtsson M, Malmqvist EL-A, Nilsson G, Sjöberg F. Laser Doppler perfusion imager (LDPI)-for the assessment of skin blood flow changes following sympathetic blocks. Acta Anaesthesiol Scand 1996;40(9):1145-1148

4 Galvin EM, Niehof S, Medina HJ, et al. Thermographic temperature measurement compared with pinprick and cold sensation in predicting the effectiveness of regional blocks. Anesth Analg 2006;102(2):598-604

5 Goldman JM, Petterson MT, Kopotic RJ, Barker SJ. Masimo signal extraction pulse oximetry. J Clin Monit Comput 2000;16(7):475-483

6 Galvin EM, Niehof S, Verbrugge SJ, et al. Peripheral flow index is a reliable and early indicator of regional block success. Anesth Analg 2006;103(1):239-243

7 Kingslin AS. Prediction of successful supraclavicular brachial plexus block using pulse oximeter perfusion index. Glob J Res Anal 2019;4:8

8 Kus A, Gurkan Y, Gormus SK, Solak M, Toker K. Usefulness of perfusion index to detect the effect of brachial plexus block. J Clin Monit Comput 2013;27(3):325-328

9 Minville V, Gendre A, Hirsch J, et al. The efficacy of skin temperature for block assessment after infraclavicular brachial plexus block. Anesth Analg 2009;108(3):1034-1036

10 Talke P, Snapir A, Huiku M. The effects of sympathectomy on finger photoplethysmography and temperature measurements in healthy subjects. Anesth Analg 2011;113(1):78-83

11 Bergek C, Zdolsek JH, Hahn RG. Non-invasive blood haemoglobin and plethysmographic variability index during brachial plexus block. Br J Anaesth 2015;114(5):812-817

12 Park SG, Lee OH, Park $\mathrm{Y}-\mathrm{H}$, et al. The changes of non-invasive hemoglobin and perfusion index of Pulse CO-oximetry during induction of general anesthesia. Korean J Anesthesiol 2015;68(4):352-357

13 Ginosar Y, Weiniger CF, Meroz Y, et al. Pulse oximeter perfusion index as an early indicator of sympathectomy after epidural anesthesia. Acta Anaesthesiol Scand 2009;53(8):1018-1026

14 Yamazaki H, Nishiyama J, Suzuki T. Use of perfusion index from pulse oximetry to determine efficacy of stellate ganglion block. Local Reg Anesth 2012;5:9-14 
15 Klodell CT, Lobato EB, Willert JL, Gravenstein N. Oximetry-derived perfusion index for intraoperative identification of successful thoracic sympathectomy. Ann Thorac Surg 2005;80(2):467-470

16 Sebastiani A, Philippi L, Boehme S, et al. Perfusion index and plethysmographic variability index in patients with interscalene nerve catheters. Can J Anaesth 2012;59(12):1095-1101

17 Abdelnasser A, Abdelhamid B, Elsonbaty A, Hasanin A, Rady A. Predicting successful supraclavicular brachial plexus block using pulse oximeter perfusion index. $\mathrm{Br}$ J Anaesth 2017;119(2):276-280 